\title{
CONTINUA WHOSE CONE AND HYPERSPACE ARE HOMEOMORPHIC
}

\author{
BY
}

SAM B. NADLER, JR.(1), (2)

\begin{abstract}
Let $X$ be a (nonempty) metric continuum. By the hyperspace of $X$ we mean $C(X)=\{A: A$ is a nonempty subcontinuum of $X\}$ with the Hausdorff metric $H$. It is determined that there are exactly eight hereditarily decomposable continua $X$ such that the cone over $X$ is homeomorphic to $C(X)$. Information about cone-to-hyperspace homeomorphisms, and about arc components for general classes of continua whose cone and hyperspace are homeomorphic is obtained. It is determined that indecomposable continua whose cone and hyperspace are homeomorphic have arcwise connected composants and, if finite-dimensional, have a strong form of the cone = hyperspace property.
\end{abstract}

1. Introduction. For each $n=1,2, \ldots$, let $R^{n}$ denote Euclidean $n$-space. We define the following special continua:

(1) $S_{0}=$ the closure of $\{(x, \sin [1 / x]): 0<x \leqslant 1\}$;

(2) $S_{1}=S^{1}=\left\{(x, y) \in R^{2}: x^{2}+y^{2}=1\right\}$;

(3) $S_{2}^{1}=S_{0}$ with the points $(0,-1)$ and $(1, \sin [1])$ identified;

(4) $S_{3}^{1}=S_{0} \cup\{(x, \sin [1 / x]):-1 \leqslant x<0\}$ with the points $(1, \sin [1])$ and $(-1, \sin [-1])$ identified;

(5) $(S P)_{1}=S^{1} \cup\left\{[1+(1 / t)] \cdot e^{i t}: t \geqslant+1\right\}$;

(6) $(S P)_{2}=(S P)_{1} \cup\left\{[1-(1 / t)] \cdot e^{i t}: t \geqslant+1\right\}$ with the points $2 e^{i \cdot 1}$ and $(0,0)$ identified;

(7) $(S P)_{3}=(S P)_{1} \cup\left\{[1+(1 / t)] \cdot e^{i t}: t \leqslant-1\right\}$ with the points $2 e^{i \cdot 1}$ and $(0,0)$ identified.

In descript terms, $S_{2}^{1}$ is the usual $\sin [1 / x]$-circle commonly called the

Presented to the Society, June 29, 1972; received by the editors July 17, 1972 and, in revised form, July 14, 1975.

AMS (MOS) subject classifications (1970). Primary 54B20; Secondary 54F20.

Key words and phrases. Chainable, circle-like, compactification, composant, decomposable continuum, dimension, Hausdorff metric, indecomposable continuum, remainder of a compactification, segment (in the sense of Kelley).

(l) This paper was completed while the author was a visitor at the University of Georgia, Athens, Georgia 30602. The author gratefully acknowledges the hospitality of the University of Georgia during his stay.

(2) This work was partially supported by Lykes Brothers Steamship Company, Inc.

- American Mathematical Society 1977 
Warsaw circle, $S_{3}^{1}$ is a particular compactification of $R^{1}$ with an arc as the remainder, $(S P)_{1}$ is a circle with a counterclockwise-moving half-line spiraling down on it, $(S P)_{2}$ is a circle with a counterclockwise-moving line spiraling down on it, and $(S P)_{3}$ is a compactification of $R^{1}$ with half of $R^{1}$ spiraling down on $S^{1}$ in a counterclockwise direction and the other half of $R^{1}$ spiraling down on $S^{1}$ in a clockwise direction. We note that all the continua except $(S P)_{3}$ are embeddable in the plane.

If $X$ is a continuum such that the cone over $X$ is homeomorphic to $C(X)$, then we will say that $X$ is a C-H continuum.

The main purpose of this paper is to prove the following result:

(1.1) THEOREM. If $X$ is an hereditarily decomposable $C$ - $H$ continuum, then $X$ is homeomorphic to one of the following eight continua: the closed unit interval $[0,1], S_{0}, S_{i}^{1}$ for $i=1,2$, or 3 , or $(S P)_{i}$ for $i=1,2$, or 3 . Furthermore, each of these eight continua are $C-H$ continua.

We prove some of this theorem in $\S \S 2$ and 3 , where we obtain other results as well [see, especially, (2.5)], and we finish the proof in $\$ 4$.

In $\$ 5$ we give some results for indecomposable C-H continua. In [14] Rogers defined a continuum $X$ to have the cone $=$ hyperspace property if and only if there is a homeomorphism $h$ from the cone over $X$ onto $C(X)$ such that $h$ takes the vertex to $X \in C(X)$ and the base onto the space of singletons. A C-H continuum may fail to have Rogers' cone $=$ hyperspace property. However, in $\$ 5$ we show among other results that if $X$ is a finite-dimensional indecomposable $\mathrm{C}-\mathrm{H}$ continuum, then any homeomorphism from the cone onto the hyperspace must take the vertex to $X$ and the base onto the space of singletons. This result should facilitate the study of such continua.

In June, 1972, I sent an abstract to the Notices [9] which announced some results on $\mathrm{C}-\mathrm{H}$ continua. Shortly thereafter I submitted a manuscript containing the results announced in [9], and others, to the Transactions. The manuscript was subsequently accepted for publication in the Transactions, modulo some revisions. In the meantime, [15] appeared and many of the results in my original manuscript were in [15]. All the results in $\$ 2$ of this paper were in my original manuscript, the principal one being (2.7) which later appeared in [15] as Theorem 4. My original proof of (2.7) is included here because there seems to be a major problem with the proof in [15]. The claim that $A^{*}$ is homeomorphic to $[0,1)$ and $A^{*}=A[15$, lines $26-27$, p. 285$]$ does not seem to be verifiable on the basis of anything said before. For example: Take $B=\left[S_{0} \backslash\{(0,1)\}\right] \cup K \cup L \cup M$ where $S_{0}$ is as in (1) above, the symbol " $\backslash$ " denotes complementation, and

$$
K=\left\{(0, y) \in R^{2}:-2 \leqslant y \leqslant-1\right\},
$$




$$
\begin{aligned}
L & =\left\{(x,-2) \in R^{2}: 0 \leqslant x \leqslant+1\right\}, \\
M & =\left\{(1, y) \in R^{2}:-2 \leqslant y \leqslant \sin [1]\right\} .
\end{aligned}
$$

Let $X=f[B] \cup Q$, where $f$ is a homeomorphism, be a compactification of $f[B]$ with an arc $Q$ as the remainder. Take $A=f[B][15$, line 21, p. 285], $a=f((0,-2))\left[15\right.$, line 22, p. 285], and $\left\{a_{i}\right\}_{i=1}^{\infty}[15$, line 23, p. 285] to be a convergent subsequence of the sequence $\{f((2 /(\pi+4 n \pi), 1))\}_{n=1}^{\infty}$. Of course $\left\{a_{i}\right\}_{i=1}^{\infty}$ must converge to a point of $Q$-the arc $Q$ "plays the role of" $h(v)$ in $[15$, p. 285]. It can now be seen that Rogers' claim that $A^{*}$ is homeomorphic to $[0,1)$ and $A^{*}=A[15$, lines $25-26$, p. 285] cannot be verified from what he did. It may be that by the term "non-cut-point" [15, line 22, p. 285] Rogers meant "does not continuumwise disconnect." But, with this new meaning, each point of $A$ is a cut point of $A$ and the trouble shifts from [15, lines 26-27, p. 285] to [15, lines $28-29$, p. 285]. I have, thus, included in $\$ 2$ my original proof of $(2.7)$; the verifications are somewhat different than those in [15].

We adopt the following notation and definitions. The letter $X$ denotes a (nonempty) metric continuum. The cone over $X$ is the decomposition space of the upper semicontinuous decomposition $(X \times[0,1]) / X \times\{1\}$, where " $\times$ " denotes cartesian product. The cone over $X$ will be denoted by $\operatorname{Cone}(X)$, its base $X \times\{0\}$ by $B(X)$, and its vertex $X \times\{1\} \in \operatorname{Cone}(X)$ by $v$. If $Y \subset X$, then when we write $\operatorname{Cone}(Y)$ we will mean "the natural subset" of $\operatorname{Cone}(X)$ given by

$$
\operatorname{Cone}(Y)=\{(x, t) \in[\operatorname{Cone}(X) \backslash\{v\}]: x \in Y\} \cup\{v\} ;
$$

also, $B(Y)=\{(x, 0) \in B(X): x \in Y\}$. The symbol $\pi$ will denote the projection $\pi$ : Cone $(X) \backslash\{v\} \rightarrow B(X)$ given by $\pi((x, t))=(x, 0)$.

The symbol $\bar{A}$ denotes the closure of $A$.

If $f: Y \rightarrow Z$ is a function, then we let $G(f) \subset[Y \times Z]$ denote the graph of $f$. If $A \subset Y$, then $f \mid A$ denotes the restriction of $f$ to $A$.

Let $Z=g\left(R^{1}\right) \cup Q, g$ a homeomorphism, be a compactification of $g\left(R^{1}\right)$ with remainder $Q$. By an end of the compactification $Z$ we mean $\bigcap_{n=1}^{\infty} \overline{g([n,+\infty))}$ or $\cap_{n=1}^{\infty} \overline{g((-\infty,-n])}$; thus, $Z$ has two ends and the ends are independent of the homeomorphism $g$ (except for possibly being switched, one for the other).

If $M \subset Y$ and $p \in Y$, then we say a continuous function $\varphi: M \times[0,1] \rightarrow Y$ is a contraction of $M$ to $p$ if and only if $\varphi((m, 0))=m$ and $\varphi((m, 1))=p$, each $m \in M$.

We refer the reader to [5] for preliminary information about the space $C(X)$. In particular, we use the word segment to mean a segment in the sense of [5, p. 24]. If $Y \subset X$ ( $Y$ not necessarily compact or connected), then $C(Y)=\{A \in C(X): A \subset Y\}$ and $\hat{Y}=\{\{y\}: y \in Y\}$, the space of single- 
tons of $Y$. We note that the function $i: Y \rightarrow \hat{Y}, i(y)=\{y\}$, is an isometry. If $x \in X$, then $X(x)=\{A \in C(X): x \in A\}$.

By an arc component of a space $Y$ we mean a maximal arcwise connected subset of $Y$. If $Y$ has no more than $n$ arc components, then we write $\alpha[Y] \leqslant n$; if $Y$ has exactly $n$ arc components, then we write $\alpha[Y]=n$. Thus, $\alpha[Y]=1$ means $Y$ is arcwise connected.

A 2-cell is a continuum homeomorphic to $\left\{(x, y): x^{2}+y^{2} \leqslant 1\right\}$. If $M$ is a $2-$ cell, then we let $\delta M$ denote the manifold boundary of $M$, i.e., $\delta M=h^{-1}\left[S^{1}\right]$ where $h$ is any homeomorphism of $M$ onto $\left\{(x, y) \in R^{2}: x^{2}+y^{2} \leqslant 1\right\}$. When we say that a point $p$ of a space $Y$ is interior to a 2-cell $M$ in $Y$ we mean that $p \in[M \backslash \delta M]$, where $\backslash$ denotes complement. In particular, then, we do not assume $M \backslash \delta M$ is open in $Y$. The following lemma will be used many times.

(1.2) LeMMA. If arcs and circles are the only nondegenerate locally connected subcontinua of $X$, then no point of $B(X)$ is interior to a 2-cell in $\operatorname{Cone}(X)$.

Proof. Let $(p, 0) \in B(X)$ and assume $(p, 0) \in \Delta$ where $\Delta$ is a 2-cell in Cone $(X)$. Choose a new 2-cell $\Delta^{\prime}$ if necessary such that $v \notin \Delta^{\prime},(p, 0) \in \Delta^{\prime}$, and such that if $(p, 0)$ were interior to $\Delta$, then $(p, 0)$ is interior to $\Delta^{\prime}$. Now, since $\pi\left[\Delta^{\prime}\right]$ is locally connected, $\pi\left[\Delta^{\prime}\right]$ is an arc or a circle (note: the formula for $\pi$ says $\pi\left[\Delta^{\prime}\right]$ is nondegenerate; otherwise $\Delta^{\prime}$ would be contained in $\pi^{-1}((p, 0))$ which is an arc). Clearly, from the formula for $\pi, \Delta^{\prime}$ $\subset$ Cone $\left(\pi\left[\Delta^{\prime}\right]\right)$. Since a point (e.g., $\left.(p, 0)\right)$ in an arc or a circle (e.g., $\left.\pi\left[\Delta^{\prime}\right]\right)$ cannot be interior to any 2-cell in the cone over the arc or circle, the result now follows. form:

One of the results announced in [9] appeared in [10, p. 242] in the following

(1.3) Dimension Theorem. If $X$ is a finite-dimensional $C-H$ continuum, then $X$ does not contain a nondegenerate hereditarily indecomposable continuum; thus, $X$ is one-dimensional.

The latter part of this result will be used many times in this paper. We mention that Rogers [15, Theorem 8] proved a much stronger result which we state as follows:

(1.4) THEOREM [15]. If $X$ is a finite-dimensional C-H continuum, then $X$ contains at most one nondegenerate indecomposable continuum.

The techniques we used to prove (1.3) do not prove (1.4), and we refer the reader to [15] for the very elegant proof of (1.4). We mention that (1.4) shows how close (1.1) and (5.7) of this paper come to completely determining all finite-dimensional $\mathrm{C}-\mathrm{H}$ continua. 
I express my sincere appreciation to B. J. Ball for several discussions with me about the material in this paper.

2. Some general results. As mentioned in the introduction all the results in this section were in my original manuscript (cf. [9]), and there is overlap with some results here and some in [15]. We give references to [15] when overlap occurs.

We begin with the following lemma. It lists two simple observations which we use several times.

(2.1) Lemma. (1) If $A$ is a subcontinuum of $X$, then $C(X) \backslash C(A)$ is an open arcwise connected subset of $C(X)$ (cf. [15, Proposition 1]).

(2) If $\alpha[X]=2$ and $h$ : Cone $(X) \stackrel{\text { onto }}{\longrightarrow} C(X)$ is a homeomorphism, then $h(v) \notin X$ and $h(v) \neq X$.

Proof. (1) Using 2.3 of [5], we see that there is a segment from any member of $C(X) \backslash C(A)$ to $X$. Hence (2.2 of [5]), $\alpha[C(X) \backslash C(A)]=1$. Since $C(A)$ is a continuum, $C(X) \backslash C(A)$ is an open subset of $C(X)$.

(2) Since $\alpha[X]=2, \alpha[\operatorname{Cone}(X) \backslash\{v\}]=2$. Thus, $\alpha[C(X) \backslash\{h(v)\}]=2$. Using 2.2 and 2.3 of [5] it is easy to see that $\alpha[C(X) \backslash\{x\}]=1$ for any $\{x\} \in X$. Hence, since $\alpha[C(X) \backslash\{h(v)\}]=2, h(v) \notin \hat{X}$. Now we show that $h(v) \neq X$. Note that:

(i) if $X$ is decomposable then, by 8.2 of [5], $\alpha[C(X) \backslash\{X\}]=1$;

(ii) if $X$ is indecomposable then, by the proof of 8.2 of [5], $C(X) \backslash\{X\}$ has uncountably many arc components (see (5.1)). By (i) and (ii) and the fact that $\alpha[C(X) \backslash\{h(v)\}]=2, h(v) \neq X$.

(2.2) THEOREM [15, P. 285, LINES 7-8]. If $X$ is an hereditarily decomposable $C$ $H$ continuum, then $\alpha[X] \leqslant 2$.

Proof. Let $A \in C(X)$. By (1) of 2.1, $\alpha[C(X) \backslash C(A)]=1$. Also, since $A$ is decomposable or $A \in \hat{X}$, we have by 8.2 of [5] that $\alpha[C(A) \backslash\{A\}]=1$. Thus, since $C(X) \backslash\{A\}=[C(X) \backslash C(A)] \cup[C(A) \backslash\{A\}]$, we have proved that $\alpha[C(X) \backslash\{A\}] \leqslant 2$. Thus, for a homeomorphism $h$ : $\operatorname{Cone}(X) \stackrel{\text { onto }}{\longrightarrow} C(X)$, $\alpha[C(X) \backslash\{h(v)\}] \leqslant 2$ and, hence, $\alpha[\operatorname{Cone}(X) \backslash\{v\}] \leqslant 2$. Therefore $\alpha[X] \leqslant 2$.

The next result shows that there is a compact nowhere dense arc component in certain C-H continua.

(2.3) THEOREM. If $X$ is an hereditarily decomposable $C$ - $H$ continuum such that $\alpha[X]=2$ and if $h: \operatorname{Cone}(X) \stackrel{\text { onto }}{\longrightarrow} C(X)$ is a homeomorphism, then $h(v)$ is an arc component of $X[15, p$. 285, line 14]; furthermore, $h(v)$ is a nowhere dense subcontinuum of $X$.

Proof. Since $\alpha[X]=2$, it follows that $\alpha[C(X) \backslash\{h(v)\}]=2$. Also, from part of the proof of (2.2), it follows that 
(2.3.1) the two arc components of $C(X) \backslash\{h(v)\}$ are $C(X) \backslash C(h(v))$ and $C(h(v)) \backslash\{h(v)\}$.

Now, let $Z_{1}$ and $Z_{2}$ denote the two arc components of $X$. Clearly $h(v) \cap Z_{i} \neq \varnothing$ for some $i$, say $i=1$. Suppose $h(v) D Z_{1}$. Let $p \in[h(v)$ $\left.\cap Z_{1}\right]$ and let $q \in\left[Z_{1} \backslash h(v)\right]$. Since $Z_{1}$ is an arc component of $X$ there is an arc $\gamma \subset \hat{X}$ from $\{p\}$ to $\{q\}$. By (2) of $(2.1), h(v) \notin \gamma$. Thus, since $\{p\}$ $\in[C(h(v)) \backslash\{h(v)\}]$ and $\{q\} \in[C(X) \backslash C(h(v))]$, we have a contradiction to (2.3.1). Hence, $h(v) \supset Z_{1}$. Now, suppose $h(v) \cap Z_{2} \neq \varnothing$. Then, the same argument as that just given shows that $h(v) \supset Z_{2}$. Thus, since $h(v) \supset Z_{1}, h(v)$ $=X$ which contradicts (2) of (2.1). Hence, $h(v) \cap Z_{2}=\varnothing$ and so $h(v)=Z_{1}$, an arc component of $X$. Note that since $Z_{1}=h(v) \in C(X), Z_{1}$ is a subcontinuum of $X$. Suppose $Z_{1}$ is not nowhere dense in $X$. Since $X=Z_{1} \cup Z_{2}$ is a continuum and $Z_{1}$ is compact, $Z_{2} \cap Z_{1} \neq \varnothing$. Furthermore, since $Z_{1}$ is not nowhere dense in $X$ and $Z_{1} \cap Z_{2}=\varnothing, \overline{Z_{2}} \triangleright Z_{1}$. Let $p \in\left[\overline{Z_{2}} \cap Z_{1}\right]$. By 2.3 of [5], there is a segment $\sigma:[0,1] \rightarrow C\left(\overline{Z_{2}}\right)$ from $\{p\}$ to $\overline{Z_{2}}$. Since $\sigma(t) \subset \overline{Z_{2}}$ for all $t \in[0,1]$ (see 2.2 of $[5]$ ) and $Z_{1} \oplus \overline{Z_{2}}, \sigma(t) \neq Z_{1}$ for any $t \in[0,1]$. Thus, $\sigma([0,1])$ is an arc in $C(X) \backslash\{h(v)\}$ such that $\sigma(0) \in[C(h(v)) \backslash\{h(v)\}]$ and $\sigma(1) \in[C(X) \backslash C(h(v))]$. This contradicts (2.3.1). Therefore, $h(v)=Z_{1}$ is nowhere dense in $X$.

(2.4) Corollary. Assume $X$ and $h$ satisfy the hypotheses of (2.3) and let $V=h(v)$. Then $h[\operatorname{Cone}(V)]=C(V)$ and $C(V)$ is nowhere dense in $C(X)$.

Proof. By (2.3), $V$ is nowhere dense in $X$. Thus, Cone $(V)$ is nowhere dense in Cone $(X)$. Hence, $h[\mathrm{Cone}(V)]$ is nowhere dense in $C(X)$. It suffices to prove that $h[\mathrm{Cone}(V)]=C(V)$. By (2.3), $V$ is an arc component of $X$. Since $\alpha[X]=2, X \backslash V=W$ is the other arc component of $X$. Hence, the arc components of $\operatorname{Cone}(X) \backslash\{v\}$ are $\operatorname{Cone}(V) \backslash\{v\}$ and $\operatorname{Cone}(W) \backslash\{v\}$. Thus, $h[$ Cone $(V) \backslash\{v\}]$ and $h[\operatorname{Cone}(W) \backslash\{v\}]$ are the arc components of $C(X) \backslash\{V\}$. Now, by (2.3.1), the arc components of $C(X) \backslash\{V\}$ are $C(X) \backslash C(V)$ and $C(V) \backslash\{V\}$. Hence, either (i) $h[\operatorname{Cone}(V) \backslash\{v\}]=C(X) \backslash C(V)$ or (ii) $h[\operatorname{Cone}(V) \backslash\{v\}]=C(V) \backslash\{V\}$. But, since $V \neq X(V$ is nowhere dense in $X)$, we have by (1) of (2.1) that $C(X) \backslash C(V)$ is a nonempty open subset of $C(X)$. Thus, if (i) held, $h[\operatorname{Cone}(V)]$ would not be nowhere dense in $C(X)$. Thus, (ii) holds and we have proved that $h[\operatorname{Cone}(V)]=C(V)$.

I originally obtained Theorem 2.5 below without excluding the possibility that some arc component of $X$ could consist of only one point. In Corollary 7 of [15], Rogers shows that no arc component of $X$ is degenerate. I use this fact below to obtain a slight improvement of my original result.

(2.5) THEOREM. If $X$ is a finite-dimensional $C$ - $H$ continuum, then

(1) any arc component of $X$ is a one-to-one continuous image of $[0,1],[0,+\infty)$, or $R^{1}$; 
(2) a compact arc component of $X$ is an arc or an arcwise connected circle-like continuum.

Proof. (1) By (1.3) $\operatorname{dim}[X]=1$. Hence (see $[4$, p. 34]), $\operatorname{dim}[\operatorname{Cone}(X)]=2$ and, therefore, $\operatorname{dim}[C(X)]=2$. Thus, by Corollary 1 of [16], $X$ is $a$-triodic. Let $L$ denote an arc component of $X$. Since $L$ contains no simple triod we have, by Theorem 3.2 of [3], that $L$ is a one-to-one continuous image of a connected linearly ordered separable space $M$ (note: the separability follows from the techniques used to prove Lemma 3.1 and Theorem 3.2 of [3]). By Corollary 7 of [15], $M$ consists of more than one point. Hence, $M$ must be homeomorphic to $[0,1],[0,+\infty)$, or $R^{1}$.

(2) Assume $L$ is a compact arc component of $X$. From the Structure Theorem for Real Curves [12, p. 9], it is easy to deduce that any compact oneto-one continuous image of $R^{1}$ must contain a triod. Thus, since $X$ is $a$-triodic, $L$ is not a one-to-one continuous image of $R^{1}$. So, assume $L$ is a (compact) one-to-one continuous image of $[0,+\infty)$. Using the Structure Theorem in [8, p. 128] and the fact that $L$ is $a$-triodic, it follows easily that $L$ is an arcwise connected circle-like continuum. This completes the proof of (2).

(2.6) THEOREM. If $X$ is a $C-H$ arcwise connected circle-like continuum and if $h: \operatorname{Cone}(X) \stackrel{\text { onto }}{\longrightarrow} C(X)$ is a homeomorphism, then $h[B(X)]=X$.

Proof. If $X$ is locally connected, then $X$ is a circle and it is easy to see that each member of $C(X) \backslash X$ is interior to a 2-cell in $C(X)$. Hence, $h[B(X)] \subset \hat{X}$ but, since any proper subcontinuum of $\hat{X}$ is an arc, it follows that $h[B(X)]$ $=X$. For the rest of the proof assume $X$ is not locally connected. Then, by Lemma 10 of [8], there is one and only one point $x_{0} \in X$ such that $X \backslash\left\{x_{0}\right\}$ is arcwise connected. Now, let

$$
\Gamma_{x_{0}}=\left\{K \in C(X): x_{0} \in K \text { and } K \text { is an } \operatorname{arc}\right\} \cup\{X\} .
$$

Using Theorem 6 of [11] it follows that each member of $C(X) \backslash\left[X \cup \Gamma_{x_{0}}\right]$ is interior to a 2-cell in $C(X)$. Hence, by (1.2), $h[B(X)] \subset\left[X \cup \Gamma_{x_{0}}\right]$. Now, it is easy to see that if $K_{1}, K_{2} \in \Gamma_{x_{0}}$, then $K_{1} \subset K_{2}$ or $K_{2} \subset K_{1}$. This implies $\Gamma_{x_{0}}$ is an arc. Furthermore, $\Gamma_{x_{0}} \cap \hat{X}=\left\{\left\{x_{0}\right\}\right\}$. Thus, it follows that the only subcontinuum of $\hat{X} \cup \Gamma_{x_{0}}$ which is homeomorphic to $X$ is $\hat{X}$. Therefore, $h[B(X)]=\hat{X}$.

(2.7) THEOREM ([15]; SEE THIRD PARAGRAPH FOLlOWING (1.1) HERE). If $X$ is an hereditarily decomposable $C-H$ continuum, then $X$ is either

(1) an arc;

(2) an arcwise connected circle-like continuum;

(3) a compactification of $[0,+\infty)$ with an arc or a circle as the remainder;

(4) a compactification of $R^{1}$ with an arc or a circle as the remainder such that each end of the compactification is equal to the remainder. 
Proof. First note that $\operatorname{dim}[X]=1$. By $(2.2), \alpha[X] \leqslant 2$. If $\alpha[X]=1$ then, by (2) of (2.5), $X$ is an arc or an arcwise connected circle-like continuum. For the rest of the proof assume $\alpha[X]=2$. Let $h$ : $\operatorname{Cone}(X) \stackrel{\text { onto }}{\longrightarrow} C(X)$ denote a homeomorphism. By (2.3), $h(v)=V$ is a compact arc component of $X$ and, by (2.4), $h[\operatorname{Cone}(V)]=C(V)$. Thus, $V$ is a C-H continuum and $\alpha[V]=1$. Hence, from what we just showed (when $\alpha[X]=1$ ), $V$ is (1) or (2). Assume $V$ is (2). Then, since $h(v)=V$, we have by (2.6) that $V$ has the cone = hyperspace property in the sense of [14]. Thus, by Theorem 7 of [14], each proper subcontinuum of $V$ is an arc. Hence, using Theorem 6 of [11], $V$ must be a circle. We have now proved that the compact arc component of $X$ is an arc or a circle. Let $Z$ denote the other arc component of $X$. Clearly $Z$ is not compact so, by (2.5), $Z$ is a one-to-one continuous image of $[0,+\infty)$ or of $R^{1}$. First, assume $Z$ is a one-to-one continuous image of $[0,+\infty)$. Since $Z$ is an open subset of $X, Z$ is locally compact. We now have that $Z$ is a locally compact noncompact one-to-one continuous image of $[0,+\infty)$. Therefore, by Theorem 7.1 of [12], $Z$ is homeomorphic to $[0,+\infty)$. To see $X$ is of type (3), we simply note that $V$ is nowhere dense in $X$ by (2.3). Next, assume $Z$ is a oneto-one continuous image of $R^{1}$. Let $f: R^{1} \stackrel{\text { onto }}{\longrightarrow} Z$ be a one-to-one continuous function, let

$$
Z_{+}=\left[\bigcap_{n=1}^{\infty} \overline{f([n,+\infty))}\right] \cap Z, \quad Z_{-}=\left[\bigcap_{n=1}^{\infty} \overline{f((-\infty,-n])}\right] \cap Z .
$$

Again, $Z$ is locally compact and noncompact. Hence, by Lemma 7.1 of [12], $Z_{+}=\varnothing$ or $Z_{-}=\varnothing$. Now, we prove the following fact:

(2.7.1) If $Z_{+}=\varnothing$, then $\cap_{n=1}^{\infty} \overline{f([n,+\infty))}=V$; similarly if $Z_{-}=\varnothing$, then $\cap_{n=1}^{\infty} \overline{f((-\infty,-n])}=V$.

PROOF OF (2.7.1). Assume $Z_{+}=\varnothing$. Then, since $X$ is compact, it follows that

$$
\left[\bigcap_{n=1}^{\infty} \overline{f([n,+\infty))}\right] \cap V \neq \varnothing .
$$

Suppose that $\cap_{n=1}^{\infty} \overline{f([n,+\infty))} \neq V$. Let $\left.p \in \overline{[f([0,+\infty))} \cap V\right]$. Since $\overline{f([0,+\infty))}$ is a continuum, there is (by 2.3 of $[5])$ a segment $\sigma:[0,1]$ $\rightarrow C(\overline{f([0,+\infty)}))$ such that $\sigma(0)=\{p\}$ and $\sigma(1)=\overline{f([0,+\infty))}$. Since $\bigcap_{n=1}^{\infty} \overline{f([n,+\infty))} \neq V$ and $Z_{+}=\varnothing$, it follows that $V \nsubseteq \overline{f([0,+\infty))}$. Hence, by (2.2) of [5], $V \notin \sigma([0,1])$. Thus, since $\sigma(0) \in[C(V) \backslash\{V\}]$ and $\sigma(1)$ $\in[C(X) \backslash C(V)]$, we have a contradiction to (2.3.1). Therefore, $\bigcap_{n=1}^{\infty} \overline{f([n,+\infty))}=V$. The other part of (2.7.1) is proved in an analogous fashion so this completes the proof of (2.7.1).

Now, since $Z_{+}=\varnothing$ or $Z_{-}=\varnothing$, assume without loss of generality that $Z_{-}=\varnothing$. We will show that $Z_{+}$is also empty. To do this suppose $Z_{+} \neq \varnothing$. Since $\operatorname{dim}[X]=1, X$ is $a$-triodic (see the beginning of the proof of (1) of (2.5)). 
Hence, $Z_{+}$is neither a single point nor an arc. Thus, by Remark 7.1 of [12], it follows that $Z_{+}=f\left(\left(-\infty, t_{0}\right]\right)$ for some $t_{0} \in R^{1}$. Now, note that since $v$ is a point of local arcwise connectivity of $\operatorname{Cone}(X), V$ is a point of local arcwise connectivity of $C(X)$. We will show that, because of our supposition that $Z_{+} \neq \varnothing$,

(*) $V$ is not a point of local arcwise connectivity of $C(X)$.

To prove (*), note first that since $V$ is an arc or a circle, $Z_{-}=\varnothing$, and $Z_{+}=f\left(\left(-\infty, t_{0}\right]\right)$, it follows that (1.2) may be applied to see that no point of $B(X)$ is interior to a 2-cell in Cone $(X)$. Using this we prove:

(2.7.2) $h[B(X)]=\hat{X}$ and $h[B(V)]=\hat{\nabla}$.

Proof of (2.7.2). Since $Z$ is a one-to-one continuous image of $R^{1}$, each member of $C(X) \backslash[\hat{X} \cup C(V)]$ is interior to a 2-cell in $C(X)$. Hence, $h[B(X)]$ $\subset[\hat{X} \cup C(V)]$. By (2.4), $h[$ Cone $(V)]=C(V)$. Thus, $h[B(Z)] \subset Z$. By $(2.3)$ $V$ is nowhere dense in $X$ and, hence, $B(Z)$ is a dense subset of $B(X)$. Therefore,

$$
h[B(X)]=\overline{h[B(Z)]} \subset \hat{Z} \subset \hat{X}
$$

and we have that $h[B(X)] \subset X$. It is easy to see, using [12, Remark 7.1, p. 72] and the fact that $V$ is nowhere dense in $X$, that no proper subcontinuum of $X$ can be homeomorphic to $X$. Therefore, $h[B(X)]=X$. Thus, $h[B(V)] \subset[C(V)$ $\cap \hat{X}]=\hat{\nabla}$. But, since $h[B(Z)] \subset \mathcal{Z}$ and $h[B(X)]=\hat{X}$, this implies that $h[B(V)]=\hat{\nabla}$. This proves (2.7.2).

Now we prove (*). Let

$$
\varepsilon=\inf \left\{H(D, V): D \in C(X) \text { and } f\left(t_{0}\right) \in D\right\} .
$$

Let $\delta>0$. Since $Z_{-}=\varnothing$, we have by $(2.7 .1)$ that $\cap_{n=1}^{\infty} \overline{f((-\infty,-n])}=V$. Thus, since $Z_{+}=f\left(\left(-\infty, t_{0}\right]\right)$, it follows that there are points of $f\left(\left[t_{0},+\infty\right)\right)$ $=Q$ arbitrarily close to points of $V$. Hence, it follows from (2.7.2) that there are points of $h^{-1}(\hat{Q})$ arbitrarily close to points of $B(V)$. For such points $(x, 0) \in h^{-1}(\hat{Q})$, the arcs $\{(x, s) \in \operatorname{Cone}(X): 0 \leqslant s \leqslant 1\}$ are as close as we like (depending only on how close $(x, 0)$ is to $B(V))$ to $\operatorname{arcs}$ in Cone $(V)$. Thus, it is now easy to see that there is a point $\left(x_{0}, s_{0}\right) \in \operatorname{Cone}(X)$ such that $H\left(h\left(\left(x_{0}, s_{0}\right)\right), V\right)<\delta$ and $h\left(\left(x_{0}, s_{0}\right)\right) \subset Q$. Let $\gamma$ be an arc in $C(X)$ from $h\left(\left(x_{0}, s_{0}\right)\right)$ to $V$. Since $Z_{+}=f\left(\left(-\infty, t_{0}\right]\right)$ and since $h\left(\left(x_{0}, s_{0}\right)\right) \subset Q$, it is easy to see that there is a $D \in \gamma$ such that $f\left(t_{0}\right) \in D$. Hence, $\operatorname{diam}[\gamma] \geqslant \varepsilon$. This proves (*). However, since (*) is false, we now conclude that $Z_{+}=\varnothing$. Therefore, since we now have that $Z_{+}$and $Z_{-}$are both empty, it follows easily that $f$ is a homeomorphism and, by (2.7.1), that $X$ is of type (4).

3. Chainable and circle-like $\mathbf{C}-\mathbf{H}$ continua. A compactification of $[0,+\infty)$ with an arc as the remainder is called an Elsa continuum, denoted E-continuum. 
An E-continuum is chainable [8, p. 126] and may be embedded in the plane in the following special way $([8$, p. 131$]$ or $[12$, p. 20$])$ :

(3.1) Lemma. If $X$ is an E-continuum, then $X$ can be embedded in the plane in such a way that the remainder is the interval $[-1,+1]$ on the $y$-axis and the rest of the continuum is the graph of a continuous function $f_{E}$ from $(0,1]$ to $[-1,+1]$.

For the rest of this paper, the letter $E$ will always denote an E-continuum embedded in the plane as in (3.1) and $f_{E}$ will always denote the continuous function in (3.1). The symbol $J$ will always denote the closed interval $[-1,+1]$ on the $y$-axis.

A particular example of an E-continuum is the familiar $\sin [1 / x]$-continuum $S_{0}$. There are uncountably many topologically different E-continua, the different topological types being a consequence of different ways $[0,+\infty)$ "patterns into" the remainder of the compactification [7, p. 184]. For example, $S_{0}$ and the two continua below in Figure 3.2 are all mutually nonhomeomorphic E-continua.
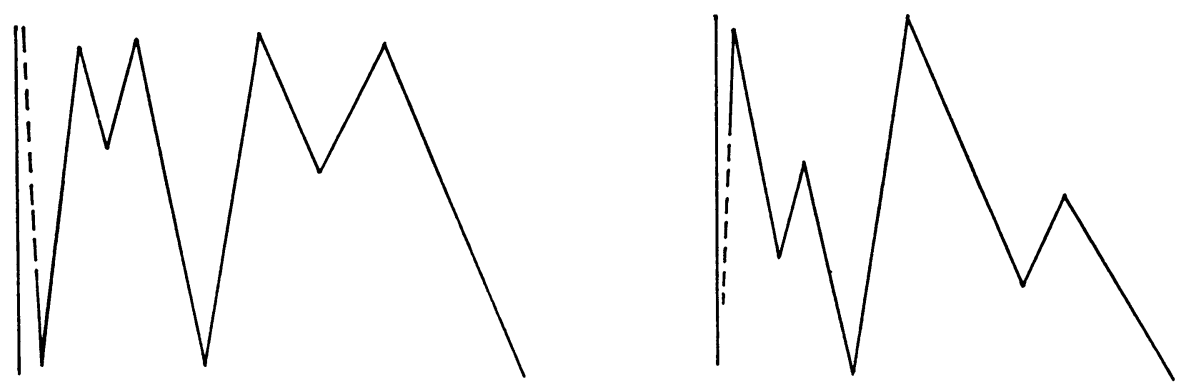

(3.2) Figure

Now we give a technical lemma which distinguishes $S_{0}$ from all other Econtinua. This lemma will be an important tool throughout most of the paper. First, we need the following notation and terminology.

(3.3) For each $i=1,2, \ldots$, let $d^{i}(1)<d^{i}(2)<\cdots<d^{i}\left(2^{i+1}+1\right)$ be an enumeration of the dyadic rationals $\left\{m \cdot 2^{-i}: m=0, \pm 1, \pm 2, \ldots, \pm 2^{i}\right\}$ in $J$, and let $C_{i}=\left\{S_{j}^{i}: j=1,2, \ldots, 2^{i+1}\right\}$ where $S_{j}^{i}=\left\{(x, y) \in R^{2}:|x| \leqslant 2^{-i}\right.$ and $\left.d^{i}(j) \leqslant y \leqslant d^{i}(j+1)\right\}$. Hence, each $C_{i}$ is a cover of $J$ by $2^{i+1}$ closed squares $S_{j}^{i}$ in the plane such that the sides of each $S_{j}^{i}$ are of length $2^{-i}$ and such that $S_{j}^{i} \cap S_{k}^{i} \neq \varnothing$ if and only if $|j-k| \leqslant 1$ (if $k=j+1$, then the intersection is along the "top" of the boundary of $S_{j}^{i}$ and the "bottom" of the boundary of $S_{k}^{i}$ ). Thus, $C_{i}$ is a special closed chain; the members of $C_{i}$ are called links. By a subchain of $C_{i}$ we mean any subcollection of $C_{i}$ consisting of consecutively indexed links.

(3.4) Now, let $g$ be a continuous function defined on a closed interval $[s, t]$ and let $C_{i}^{\prime}=\left\{S_{j}^{i}: k_{1} \leqslant j \leqslant k_{2}\right\}$ be a subchain of $C_{i}$. We say that $G(g)$ passes 
through $C_{i}^{\prime}$ at least three times if and only if

(3.4.1) $G(g) \subset \cup C_{i}^{\prime}$;

(3.4.2) there exist $r_{1}$ and $r_{2}, s<r_{1}<r_{2}<t$, such that either $(s, g(s))$, $\left(r_{2}, g\left(r_{2}\right)\right) \in S_{k_{1}}^{i}$ and $\left(r_{1}, g\left(r_{1}\right)\right),(t, g(t)) \in S_{k_{2}}^{i}$ or $(s, g(s)),\left(r_{2}, g\left(r_{2}\right)\right) \in S_{k_{2}}^{i}$ and $\left(r_{1}, g\left(r_{1}\right)\right),(t, g(t)) \in S_{k_{1}}^{i}$.

(3.5) LeMma. If $E$ is not homeomorphic to $S_{0}$, then there exist $a, b \in J$, with $a<b$ and $\{a, b\} \neq\{-1,+1\}$, satisfying: for each $i=1,2, \ldots$, there is $a$ subchain $C_{i}^{\prime}=\left\{S_{j}^{i}: k_{1}^{i} \leqslant j \leqslant k_{2}^{i}\right\}$ of $C_{i}$ such that

(3.5.1) $a \in S_{k j}^{i}$ and $b \in S_{k i}^{i}$;

(3.5.2) there exists a closed interval $\left[s_{i}, t_{i}\right]$ such that $G\left(f_{E} \mid\left[s_{i}, t_{i}\right]\right)$ passes through $C_{i}^{\prime}$ at least three times.

Proof. It is easy to see, using the fact that $J \subset \overline{G\left(f_{E}\right)}$, that (3.5.1) and (3.5.2) hold for $a=-1$ and $b=+1$ (with $C_{i}^{\prime}=C_{i}$ ). Now, assume

(*) $a=-1$ and $b=+1$ are the only choices of distinct points of $J$ such that (3.5.1) and (3.5.2) hold for each $i$.

We define points $p_{n}$ as follows: Let $p_{1}=1$.u.b. $\left\{p \in(0,1]:\left(p, f_{E}(p)\right)\right.$ $\left.\in S_{1}^{1}\right\}, p_{2}=1$.u.b. $\left\{p \in\left(0, p_{1}\right]:\left(p, f_{E}(p)\right) \in S_{4}^{1}\right\}, p_{3}=1$.u.b. $\left\{p \in\left(0, p_{2}\right]:\right.$ $\left.\left(p, f_{E}(p)\right) \in S_{1}^{1}\right\}, \ldots$ continuing until after defining finitely many points $p_{n}$, we obtain a point $p_{n_{1}}$ such that $\left(p_{n_{1}}, f_{E}\left(p_{n_{1}}\right) \in S_{1}^{2}\right.$. Then let

$$
\begin{aligned}
& p_{n_{1}+1}=1 . \text { u.b. }\left\{p \in\left(0, p_{n_{1}}\right]:\left(p, f_{E}(p)\right) \in S_{8}^{2}\right\}, \\
& p_{n_{1}+2}=1 . \text { u.b. }\left\{p \in\left(0, p_{n_{1}+1}\right]:\left(p, f_{E}(p)\right) \in S_{1}^{2}\right\}, \ldots
\end{aligned}
$$

continuing until, after defining finitely many points $p_{n}$, we obtain a point $p_{n_{2}}$ such that $\left(p_{n_{2}}, f_{E}\left(p_{n_{2}}\right)\right) \in S_{1}^{3}$. Then continue by picking points $p_{n}$ with respect to $S_{1}^{3}$ and $S_{16}^{3}$ until a point $p_{n_{3}}$ is obtain such that $\left(p_{n_{3}}, f_{E}\left(p_{n_{3}}\right)\right) \in S_{1}^{4}$. Continuing in this fashion we obtain a sequence $p_{1}>p_{2}>\cdots>p_{n}>\cdots$, which evidently converges to zero.

Now, it follows from $(*)$ that:

(\#) Given $i$, there exists $N(i)$ such that if $n \geqslant N(i)$ then, whenever $p_{n+1} \leqslant s \leqslant t \leqslant p_{n}$ and $G\left(f_{E} \mid[s, t]\right)$ passes through a subchain $C_{i}^{\prime}$ of $C_{i}$ at least three times, $C_{i}^{\prime}$ has at most two links or $C_{i}^{\prime}=C_{i}$.

For each $n=1,2, \ldots$, let $\lambda_{n}$ be the convex segment in the plane from $\left(p_{n}, f_{E}\left(p_{n}\right)\right)$ to $\left(p_{n+1}, f_{E}\left(p_{n+1}\right)\right)$. Let $E_{0}=J \cup\left[\cup_{n=1}^{\infty} \lambda_{n}\right]$. It is easy to see from the way the sequence $\left\{p_{n}\right\}_{n=1}^{\infty}$ was constructed that $\left(p_{2 n+1}, f_{E}\left(p_{2 n+1}\right)\right) \rightarrow(0$, $-1)$ and $\left(p_{2 n}, f_{E}\left(p_{2 n}\right)\right) \rightarrow(0,+1)$ as $n \rightarrow \infty$. It follows from this that $E_{0}$ is homeomorphic to $S_{0}$. Now, using (\#), it can be seen that there exists a sequence $\left\{h_{n}\right\}_{n=1}^{\infty}$ of homeomorphisms,

$$
h_{n}: \lambda_{n} \stackrel{\text { onto }}{\longrightarrow} G\left(f_{E} \|\left[p_{n}, p_{n+1}\right]\right),
$$


such that, for each $n, h_{n}\left(\left(p_{n}, f_{E}\left(p_{n}\right)\right)\right)=\left(p_{n}, f_{E}\left(p_{n}\right)\right)$, and such that $\sup \left\{d\left(h_{n}(x), x\right): x \in \lambda_{n}\right\} \rightarrow 0$ as $n \rightarrow \infty$ (where $d$ denotes distance in the plane). Thus, the function

$$
h: E_{0} \stackrel{\text { onto }}{\longrightarrow} E \backslash G\left(f_{E} \mid\left(p_{1}, 1\right]\right)
$$

defined by

$$
\begin{aligned}
h(x) & =h_{n}(x) & & \text { if } x \in \lambda_{n}, \\
& =x & & \text { if } x \in J \text { is continuous, }
\end{aligned}
$$

and hence a homeomorphism.

Next, we have the following lemma which gives information about the behavior of homeomorphisms between the cone and hyperspace of an Econtinuum.

(3.6) Lemma. If $h$ : Cone $(E) \stackrel{\text { onto }}{\longrightarrow} C(E)$ is a homeomorphism, then

(1) $h(v)=J$

(2) $h[\operatorname{Cone}(J)]=C(J)$;

(3) $h[B(J)]=\hat{\jmath}$.

PRoof. From (2.3) we have (1) and from (2.4) we have (2). To prove (3), let $\Gamma_{1}=\{K \in C(J):-1 \in K$ or $+1 \in K\}$,

$\Gamma_{2}=\left\{K \in C(E):\left(1, f_{E}(1)\right) \in K\right\}$, and

$\Gamma_{3}=\{K \in C(E): K \supset J\}$.

Let $\Gamma \in \hat{E} \cup\left[\cup_{i=1}^{3} \Gamma_{i}\right]$. It is easy to see that any member of $C(E) \backslash \Gamma$ is interior to a 2-cell in $C(E)$. Hence, by (1.2) $h[B(E)] \subset \Gamma$. Now, observe that

(i) $\Gamma_{1} \cup \mathcal{J}$ is a circle;

(ii) $\Gamma_{2} \cup \Gamma_{3}$ is an arc with endpoints $J$ and $\left\{\left(1, f_{E}(1)\right)\right\}$;

(iii) $\Gamma_{2} \cup \Gamma_{3} \cup \hat{E}$ is a topological copy of $E$, the remainder corresponding to j.

It follows that $h[B(J)]=\boldsymbol{\jmath}$.

(3.7) LeMma. If $E$ is a $C-H$ continuum, then there is a contraction $\varphi: \hat{E} \times[0,1]$ $\rightarrow C(E)$ of $\hat{E}$ to $J$ such that $\varphi$ is one-to-one on $\hat{J} \times[0,1)$.

Proof. Let $h$ : Cone $(E) \stackrel{\text { onto }}{\longrightarrow} C(E)$ be a homeomorphism and define $\lambda$ : $\operatorname{Cone}(E) \times[0,1] \rightarrow \operatorname{Cone}(E)$ by $\lambda[((x, t), s)]=(x,(1-s) t+s)$ for each $(x, t)$ $\in \operatorname{Cone}(E)$ and $s \in[0,1]$. Now, define $\varphi: \hat{E} \times[0,1] \rightarrow C(E)$ by $\varphi((\{x\}, s))$ $=h \circ \lambda\left[\left(h^{-1}(\{x\}), s\right)\right]$ for each $\{x\} \in \hat{E}$ and $s \in[0,1]$. It is easy to verify that $\varphi$ is a contraction. Now, let $\left(\left\{x_{i}\right\}, s_{i}\right) \in \mathcal{J} \times[0,1)$ for $i \in\{1,2\}$. By (3) of (3.6), $h^{-1}\left(\left\{x_{i}\right\}\right) \in B(J)$ so let $\left(y_{i}, 0\right)=h^{-1}\left(\left\{x_{i}\right\}\right)$ for each $i \in\{1,2\}$ (as we will see, the important fact here is that the second coordinate is zero). Assume that $\varphi\left(\left(\left\{x_{1}\right\}, s_{1}\right)\right)=\varphi\left(\left(\left\{x_{2}\right\}, s_{2}\right)\right)$. Then, since $h$ is one-to-one, $\lambda\left[\left(\left(y_{1}, 0\right), s_{1}\right)\right]$ $=\lambda\left[\left(\left(y_{2}, 0\right), s_{2}\right)\right]$ and the formula for $\lambda$ gives $\left(y_{1}, s_{1}\right)=\left(y_{2}, s_{2}\right)$. Therefore 
$s_{1}=s_{2}$ and, since $s_{i} \neq 1(i=1,2),, y_{1}=y_{2}$. This completes the proof that $\varphi$ is one-to-one on $\delta \times[0,1)$. To see that $\varphi$ contracts $\hat{E}$ to $J$, let $\{x\} \in \hat{E}$. Then, from the formula for $\lambda, \lambda\left[\left(h^{-1}(\{x\}), 1\right)\right]=v$. Therefore, by (1) of (3.6) and the formula for $\varphi, \varphi((\{x\}, 1))=h(v)=J$.

(3.7.1) Remark. The contraction $\varphi$ of (3.7) has other properties not mentioned in (3.7) because they will not be used. For example: (i) if $\varphi((\{x\}, s))=J$, then $s=1$, (ii) $\varphi(\hat{J} \times[0,1]) \subset C(J)$, and (iii) $\varphi([\hat{E} \backslash \hat{J}] \times[0,1))$ $\subset[C(E) \backslash C(J)]$.

The following lemma is valid for any two continuous functions from any two continua into the hyperspace of an arc, but we state it in the form we will use here.

(3.8) Lemma. Let $a \leqslant b$ and let

$$
\alpha:\left[0, w_{1}\right] \rightarrow C([a, b]) \text { and } \beta:\left[0, w_{2}\right] \rightarrow C([a, b])
$$

be continuous functions such that $\alpha(0)=\{a\}, b \in \alpha\left(w_{1}\right), \beta(0)=\{b\}$, and $a$ $\in \beta\left(w_{2}\right)$. Then there exist $s \in\left[0, w_{1}\right]$ and $t \in\left[0, w_{2}\right]$ such that $\alpha(s)=\beta(t)$.

Proof. If $a<b, C([a, b])$ is a 2-cell. The following facts about $C([a, b])$ are easy to verify:

(i) $C_{a}([a, b])=\{K \in C([a, b]): a \in K\}$ is an arc lying in the boundary of $C([a, b])$ with endpoints $\{a\}$ and $[a, b]$.

(ii) $C_{b}([a, b])=\{K \in C([a, b]): b \in K\}$ is an arc lying in the boundary of $C([a, b])$ with endpoints $\{b\}$ and $[a, b]$.

(iii) $C_{a}([a, b]) \cap C_{b}([a, b])=\{[a, b]\}$.

(iv) The simple closed curve $S$ which is the boundary of $C([a, b])$ is given by

$$
S=C_{a}([a, b]) \cup C_{b}([a, b]) \cup \widehat{[a, b]} .
$$

Now, let $\Gamma=\alpha\left(\left[0, w_{1}\right]\right)$ and let $\Lambda=\beta\left(\left[0, w_{2}\right]\right)$. Since $\alpha(0)=\{a\}$ and $\beta(0)$ $=\{b\}$, we may assume for the purpose of proof that $\{b\} \notin \Gamma$ and $\{a\} \notin \Lambda$. Note that $\Gamma$ is a subcontinuum of $C([a, b])$ intersecting $S$ at $\{a\}$ and $\alpha\left(w_{1}\right)$ and $\Lambda$ is a subcontinuum of $C([a, b])$ intersecting $S$ at $\{b\}$ and $\beta\left(w_{2}\right)$. Furthermore, by (i) through (iv), $\left\{\{a\}, \alpha\left(w_{1}\right)\right\}$ disconnects $S$ between $\{b\}$ and $\beta\left(w_{2}\right)$. Hence, by facts about the topology of the plane [6], $\Gamma \cap \Lambda \neq \varnothing$ (if $\Gamma \cap \Lambda=\varnothing$, then one can obtain two disjoint arcs $\gamma$ and $\lambda$ in $C([a, b])$ such that $\gamma \cap S$ $=\left\{\{a\}, \alpha\left(w_{1}\right)\right\}$ and $\lambda \cap S=\left\{\{b\}, \beta\left(w_{2}\right)\right\}$; then, the $\theta$-curve theorem [6, p. 511] is contradicted). This proves (3.8).

(3.9) THEOREM. If there is a contraction $\varphi: \hat{E} \times[0,1] \rightarrow C(E)$ of $\hat{E}$ to $J$ such that $\varphi$ is one-to-one on $\mathcal{J} \times[0,1)$, then $E$ is homeomorphic to $S_{0}$.

Proof. Let $\varphi: \hat{E} \times[0,1] \rightarrow C(E)$ be a contraction of $\hat{E}$ to $J$ such that $\varphi$ is one-to-one on $J \times[0,1)$. Let $a, b \in J$, with $a<b$, such that (3.5.1) and (3.5.2) 
hold, and let $\left[s_{i}, t_{i}\right]$ be as in (3.5.2). For each $i=1,2, \ldots$, let $r_{1}^{i}<r_{2}^{i}$ satisfy (3.4.2) for $g=f_{E} \mid\left[s_{i}, t_{i}\right]$. It is easy to see that $G\left(f_{E} \|\left[r_{1}^{i}, r_{2}^{i}\right]\right) \rightarrow[a, b]$ as $i \rightarrow+\infty$. Also, the sequences $\left\{r_{1}^{i}\right\}_{i=1}^{\infty}$ and $\left\{r_{2}^{i}\right\}_{i=1}^{\infty}$ converge, one to $a$ and the other to $b$. Without loss of generality we assume $r_{1}^{i} \rightarrow a$ and $r_{2}^{i} \rightarrow b$ as $i \rightarrow+\infty$. Now, there exist $w_{1}^{i}, w_{2}^{i} \in[0,1]$ such that, for each $i=1,2, \ldots, \varphi\left(\left(\left\{r_{1}^{i}\right\}, w_{1}^{i}\right)\right) \cap S_{k_{2}}^{i}$ $\neq \varnothing, \varphi\left(\left(\left\{r_{2}^{i}\right\}, w_{2}^{i}\right)\right) \cap S_{k_{1}}^{i} \neq \varnothing$, and $\varphi\left(\left(\left\{r_{j}^{i}\right\}, w\right)\right) \subset \cup C_{i}^{\prime}$ whenever $j=1,2$ and $0 \leqslant w \leqslant w_{j}^{i}$. It now follows from convergence considerations that there exist $w_{1}, w_{2} \in[0,1]$ such that $b \in \varphi\left(\left(\{a\}, w_{1}\right)\right), a \in \varphi((\{b\}, w)), \varphi((\{a\}, w))$ $\in C([a, b])$ for all $0 \leqslant w \leqslant w_{1}$, and $\varphi((\{b\}, w)) \in C([a, b])$ for all $0 \leqslant w$ $\leqslant w_{2}$. Therefore, since $\varphi((\{a\}, 0))=\{a\}$ and $\varphi((\{b\}, 0))=\{b\}$, we have by $(3.8)$ that there exist $s \in\left[0, w_{1}\right]$ and $t \in\left[0, w_{2}\right]$ such that $\varphi((\{a\}, s))=\varphi((\{b\}, t))$. Thus, since $a \neq b$ and $\varphi$ is one-to-one on $\hat{J} \times[0,1)$, it follows that $s=1$ or $t=1$. Hence, since $\varphi$ contracts $\hat{E}$ to $J$, it now follows that $[a, b]=J$. Therefore, $a=-1$ and $b=+1$. From what we have shown, the theorem now follows from (3.5).

The next two theorems are the main results of this section.

(3.10) THEOREM. If $X$ is an hereditarily decomposable $C$ - $H$ chainable continuum, then $X$ is either an arc or $X$ is homeomorphic to $S_{0}$.

Proof. By (2.2), $\alpha[X] \leqslant 2$. If $\alpha[X]=1$, then $X$ must be an arc (see [11] for a simple proof of this well-known fact). So, assume $\alpha[X]=2$. Then by Theorem 1 of [7] and the second part of (2.3) above, $X$ is an E-continuum. The result now follows from (3.7) and (3.9).

(3.10.1) REMARK. Another proof of (3.10) can be given using (2.7) in place of (2.2) and (2.3) and then using (3.7) and (3.9). However, in view of the complicated nature of the proof of (2.7), we believe the proof given above is the simplest and "most basic".

(3.11) THEOREM. If $X$ is an hereditarily decomposable $C$ - $H$ circle-like continuum, then $X$ is homeomorphic to $S_{i}^{1}$ for some $i=1,2,3$.

Proof. Let $h$ : Cone $(X) \stackrel{\text { onto }}{\longrightarrow} C(X)$ be a homeomorphism. By (2.2), $\alpha[X]$ $\leqslant 2$.

Case I: $\alpha[X]=1$. Assume $X$ is not a circle. Then by Theorem 6 of [11] and Theorem 1 of [7], $X$ is the union of an E-continuum and an arc which intersect only in opposite endpoints [1] of the E-continuum. For convenience we will consider $X$ to be in the plane so that $X=E \cup P$ where $E$ is as in (3.1) and $P$ is the polygonal arc from $(0,-1)$ to $\left(1, f_{E}(1)\right)$ with corners at $(0,-2)$ and $(1,-2)$. Such an embedding exists as a consequence of $[12$, p. 43]. Now, note the following two properties of $h$ :

(3.11.1) $h(v)=J$. 
Proof of (3.11.1). It is easy to see that $\Lambda=[C(X) \backslash C(J)] \cup\{J\}$ is precisely the set of points at which $C(X)$ is locally arcwise connected. Hence, $h(v) \in \Lambda$. Now, furthermore, each member of $C(X) \backslash[C(J) \cup \hat{X}]$ is interior to a 2-cell in $C(X)$. Thus, $h(v) \in[C(J) \cup X]$. We can now conclude that $h(v)=J$.

(3.11.2) $h[B(J)]=\mathcal{J}$.

Proof of (3.11.2). By (2.6), $h[B(X)]=\not$. Thus, since $B(J)$ [resp., $J]$ is precisely the set of points at which $B(X)$ [resp., $\hat{X}$ ] is not locally connected, we must have $h[B(J)]=\boldsymbol{J}$.

Now, define $\lambda$ and $\varphi$ in the same way as in (3.7), $\varphi: X \times[0,1] \rightarrow C(X)$. A simple modification of the proof of (3.7), using (3.11.1) and (3.11.2) in place of (1) and (3) of (3.6), gives us that $\varphi$ is a contraction of $X$ to $J$ such that $\varphi$ is oneto-one on $j \times[0,1)$. The proof of (3.9) may now be easily modified so that we can conclude that $E$ is homeomorphic to $S_{0}$. Thus, since $X=E \cup P$ and $P \cap E=\left\{(0,-1),\left(1, f_{E}(1)\right)\right\}, X$ is homeomorphic to $S_{2}^{1}$.

Case II: $\alpha[X]=2$. By (2.7) and the fact that each proper subcontinuum of a circle-like continuum is chainable, it follows that $X$ must be a compactification of $R^{1}$ with an arc $A$ as the remainder such that each end of the compactification is equal to $A$. Using techniques in the proof of Lemma 11 of [8, p. 131] or of Lemma 5.1 of [12, p. 20], it is easy to obtain an embedding of $X$ in the plane so that

(i) $A$ corresponds to $J$ and

(ii) $X \backslash A$ corresponds to $G_{1} \cup G_{2} \cup P$, where $G_{1}$ [resp., $G_{2}$ ] is the graph of a continuous function $g_{1}:(0,1] \rightarrow J$ [resp., $g_{2}:[-1,0) \rightarrow J$ ] and $P$ is the polygonal arc from $\left(-1, g_{2}(-1)\right)$ to $\left(1, g_{1}(1)\right)$ with corners at $(-1,-2)$ and $(1,-2)$.

Now, a proof similar to that given for Case I shows that $X$ is homeomorphic to $S_{3}^{1}$.

4. Completion of the proof of (1.1). In $\S 3$ we proved that an hereditarily decomposable chainable or circle-like C-H continuum must be homeomorphic to [0,1], $S_{0}$, or $S_{i}^{1}$ for some $i=1,2$, or 3 . In this section we show that any other hereditarily decomposable C-H continuum must be homeomorphic to $(S P)_{i}$ for some $i=1,2$, or 3 .

(4.1) Lemma. If $X$ is an hereditarily decomposable $C-H$ continuum which is neither chainable nor circle-like, then $X$ is either

(4.1.1) a compactification of $[0,+\infty)$ with a circle $\Sigma$ as the remainder, or

(4.1.2) a compactification of $R^{1}$ with a circle $\Sigma$ as the remainder such that each end of the compactification is equal to $\Sigma$.

Proof. The continuum $X$ is neither (1) nor (2) of (2.7). A continuum satisfying (3) of (2.7) with an arc as remainder is chainable [8, p. 126]. It is easy to see that a continuum satisfying (4) of (2.7) with an arc as remainder is 
circle-like. The lemma now follows using (2.7).

A continuum (not necessarily a C-H continuum) satisfying (4.1.i) will be called a (4.1.i)-continuum, $i=1$ or 2 .

(4.2) Lemma. Let $X$ be a (4.1. $i$ )-continuum, $i=1$ or 2 . If $h$ : $\operatorname{Cone}(X)$ $\stackrel{\text { onto }}{\longrightarrow} C(X)$ is a homeomorphism, then

(1) $h(v)=\Sigma$;

(2) $h[\operatorname{Cone}(\Sigma)]=C(\Sigma)$

(3) $h[B(\Sigma)]=\hat{\Sigma}$.

Proof. From (2.3) we have (1) and thus, from (2.4), we have (2). From (2) and the fact that $\hat{\Sigma}$ consists of exactly those members of $C(\Sigma)$ which are not interior to a 2-cell in $C(\Sigma)$, (3) follows using (1.2).

(4.3) Lemma. If $X$ is a $C-H$ (4.1. $i)$-continuum $(i=1$ or 2$)$, then there is a contraction $\varphi: \hat{X} \times[0,1] \rightarrow C(X)$ of $\hat{X}$ to $\Sigma$ such that $\varphi$ is one-to-one on $\hat{\Sigma} \times[0,1)$.

Proof. Using (4.2) instead of (3.6), the proof can be done in a similar fashion to the proof of (3.7).

For each $i=1,2, \ldots$, let $D_{i}=\left\{A_{j}^{i}: j=1,2, \ldots, 2^{i+1}\right\}$ where, using polar coordinates,

$$
\begin{aligned}
A_{j}^{i}=\left\{(r, \theta) \in R^{2}: 1-2^{-i} \leqslant r \leqslant 1+2^{-i}\right. \text { and } & \\
& \left.(j-1) \cdot \pi \cdot 2^{-i} \leqslant \theta \leqslant j \cdot \pi \cdot 2^{-i}\right\} .
\end{aligned}
$$

Hence, each $D_{i}$ is a cover of $S^{1}$ by $2^{i+1}$ closed annular sectors $A_{j}^{i}$ in the plane. Note that $A_{j}^{i} \cap A_{k}^{i} \neq \varnothing$ if and only if $|j-k| \leqslant 1$ or $j, k \in\left\{1,2^{i+1}\right\}$ (i.e., $D_{i}$ is a special circular chain). The members of $D_{i}$ will be called links.

The following lemma is a special case of Lemma 5.6 of [12] (also see [13]).

(4.4) LemMa. Any (4.1.1)-continuum can be embedded in the plane so that the remainder is the standard circle $S^{1}$.

From now on when we write (4.1.1)-continuum we will mean the (4.1.1)continuum is embedded in the plane as in (4.4).

The following lemma distinguishes $(S P)_{1}$ from all other (4.1.1)-continua, and can be proved with methods analogous to those used to prove (3.5).

(4.5) LeMMA. Let $X$ be a (4.1.1)-continuum and let $\rho:[0,+\infty) \stackrel{\text { onto }}{\longrightarrow} X \backslash S^{1}$ be a homeomorphism. Assume that: Given $i$ there exists $r(i) \in[0,+\infty)$ such that if $\{\rho(s), \rho(t)\} \subset A_{k}^{i}$ (some $k$ ) for $t \geqslant s \geqslant r(i)$, then $\rho([s, t]) \cap A_{j}^{i} \neq \varnothing$ for all $j=1,2, \ldots, 2^{i+1}$ or $\rho([s, t])$ is contained in the union of three links of $D_{i}$. Then $X$ is homeomorphic to $(S P)_{1}$. 
(4.6) LEMMA. If $X$ is a (4.1.1)-continuum such that there is a contraction $\varphi: X \times[0,1] \rightarrow C(X)$ of $X$ to $S^{1}$ such that $\varphi$ is one-to-one on $\hat{S}^{1} \times[0,1)$, then $X$ is homeomorphic to $(S P)_{1}$.

Proof. Assume $X$ is a (4.1.1)-continuum and that $\varphi: X \times[0,1] \rightarrow C(X)$ is a contraction of $X$ to $S^{1}$ such that $\varphi$ is one-to-one on $S^{1} \times[0,1)$. Suppose $X$ is not homeomorphic to $(S P)_{1}$. Then there exists $i$ such that the assumption in (4.5) is violated for $i$. This means there exist $s$ and $t$, with $s<t$ as large as we like, such that each of the following holds:

(1) $\{\rho(s), \rho(t)\} \subset A_{k(s, t)}^{i}$ for some $k(s, t)$;

(2) $\rho([s, t]) \cap A_{j(s, t)}^{i}=\varnothing$ for some $j(s, t)$;

(3) $\rho([s, t])$ is not contained in the union of any three links of $D_{i}$.

Let $\langle i, s, t\rangle$ denote the statement "(1) through (3) hold for $i, s$, and $t$."

We produce three sequences $\left\{s_{n}\right\}_{n=1}^{\infty},\left\{t_{n}\right\}_{n=1}^{\infty}$, and $\left\{u_{n}\right\}_{n=1}^{\infty}$ as follows.

Let $M_{1}=\{s \in[0,+\infty)$ : there exists $t>s$ such that $\langle i, s, t\rangle$ holds $\}$ and let $s_{1}^{\prime}=$ g.l.b. $\left[M_{1}\right]$. Let $s_{1} \in M_{1}$ such that $\rho\left(\left[s_{1}^{\prime}, s_{1}\right]\right)$ is contained in the union of the (at most two) links of $D_{i}$ to which $\rho\left(s_{1}^{\prime}\right)$ belongs. Now, let $N_{1}=\{t$ $>s_{1}:\left\langle i, s_{1}, t\right\rangle$ holds $\}$ and note that, since $s_{1} \in M_{1}, N_{1} \neq \varnothing$. Let $t_{1}^{\prime}$ $=$ g.l.b. $\left[N_{1}\right]$. Choose $t_{1} \in N_{1}$ such that $\rho\left(\left[t_{1}^{\prime}, t_{1}\right]\right)$ is contained in the union of the (at most two) links of $D_{i}$ to which $\rho\left(t_{1}^{\prime}\right)$ belongs. Since $t_{1} \in N_{1},\left\langle i, s_{1}, t_{1}\right\rangle$ holds. Now, let $\beta: X \rightarrow S^{1}$ denote radial projection, i.e., $\beta(x)=x /|x|$ for each $x \in X$ where || denotes the distance from $x$ to the origin $(0,0)$ [Remark: we are assuming $(0,0) \notin X$ as we may without loss of generality]. From the fact that $\left\langle i, s_{1}, t_{1}\right\rangle$ holds it follows easily that $\beta\left[\rho\left(\left[s_{1}, t_{1}\right]\right)\right]$ is an arc $\gamma_{1}$ in $S^{1}$ such that one of the endpoints of $\gamma_{1}$ is of the form $\beta\left[\rho\left(u_{1}\right)\right], s_{1}<u_{1}<t_{1}$, where $\rho\left(u_{1}\right)$ is not in the union of the (at most three) links of $D_{i}$ which intersect $\left\{\rho\left(s_{1}\right), \rho\left(t_{1}\right)\right\}$. Now, let $M_{2}=\left\{s \in\left[u_{1},+\infty\right)\right.$ : there exists $t>s$ such that $\langle i, s, t\rangle$ holds $\}$ and let $s_{2}^{\prime}=$ g.l.b. $\left[M_{2}\right]$. Let $s_{2} \in M_{2}$ such that $\rho\left(\left[s_{2}^{\prime}, s_{2}\right]\right)$ is contained in the union of the (at most two) links of $D_{i}$ to which $\rho\left(s_{2}^{\prime}\right)$ belongs. Let $N_{2}=\{t$ $>s_{2}:\left\langle i, s_{2}, t\right\rangle$ holds $\}$ and let $t_{2}^{\prime}=$ g.l.b. $\left[N_{2}\right]$. Choose $t_{2} \in N_{2}$ such that $\rho\left(\left[t_{2}^{\prime}, t_{2}\right]\right)$ is contained in the union of the (at most two) links of $D_{i}$ to which $\rho\left(t_{2}^{\prime}\right)$ belongs. Since $t_{2} \in N_{2},\left\langle i, s_{2}, t_{2}\right\rangle$ holds. Again using $\beta$ we find $u_{2}$ as above and use it to define $M_{3}$, etc. In this way we produce the three sequences $\left\{s_{n}\right\}_{n=1}^{\infty},\left\{t_{n}\right\}_{n=1}^{\infty}$ and $\left\{u_{n}\right\}_{n=1}^{\infty}$.

Intuitively $\rho\left(\left[s_{n}, t_{n}\right]\right)$ travels part of the way around $S^{1}$ twice, first going essentially in one direction as the parameter goes from $s_{n}$ to $u_{n}$ and then essentially in the other direction as the parameter goes from $u_{n}$ to $t_{n}$. If the essential direction for $\rho\left(\left[s_{n}, u_{n}\right]\right)$ is clockwise [resp. counterclockwise], then the essential direction for $\rho\left(\left[s_{n+1}, u_{n+1}\right]\right)$ is counterclockwise [resp. clockwise].

By "adjusting" $s_{n}$ and $t_{n}$ and going to subsequences if necessary, we can assume $\left\{\rho\left(\left[s_{n}, t_{n}\right]\right) \cup \rho\left(\left[s_{n+1}, t_{n+1}\right]\right)\right\}_{n=1}^{\infty}$ converges to an arc $\gamma \subset S^{1}$, $\left\{\rho\left(u_{2 n-1}\right)\right\}_{n=1}^{\infty}$ converges, and $\left\{\rho\left(u_{2 n}\right)\right\}_{n=1}^{\infty}$ converges. Then, it follows from the 
fact that $C(X)$ is contractible that the sequences $\left\{\rho\left(u_{2 n-1}\right)\right\}_{n=1}^{\infty}$ and $\left\{\rho\left(u_{2 n}\right)\right\}_{n=1}^{\infty}$ converge to different points $p$ and $q$ of $\gamma$ (see Remark 4.6.1 below). An argument similar to that given in the proof of (3.9) shows that $\varphi\left(\{p\}, z_{1}\right)$ $=\varphi\left(\{q\}, z_{2}\right) \subset \gamma$. Hence, since $\varphi$ contracts $\hat{X}$ to $S^{1}, z_{1} \neq 1 \neq z_{2}$. This contradicts the one-to-oneness of $\varphi$ on $\hat{S}^{1} \times[0,1)$.

(4.6.1) REMARK. Let $X$ be the continuum obtained from $S_{0}$ by identifying $(0,-1)$ and $(0,+1)$. Then $C(X)$ is not contractible. This is a specific illustration of the situation where $\left\{\rho\left(u_{2 n-1}\right)\right\}_{n=1}^{\infty}$ and $\left\{\rho\left(u_{2 n}\right)\right\}_{n=1}^{\infty}$ converge to the same point (in this case, the "identified" point).

(4.7) THEOREM. If $X$ is a $C$-H (4.1.1)-continuum, then $X$ is homeomorphic to $(S P)_{1}$.

Proof. The result follows from (4.3) and (4.6).

(4.8) Lemma. Let $X$ be a $C-H$ (4.1.2)-continuum, let $\theta: R^{1} \stackrel{\text { onto }}{\longrightarrow} X \backslash \Sigma$ be a homeomorphism, let $X_{+}=\overline{\theta([+1,+\infty))}$, and let $X_{-}=\overline{\theta((-\infty,-1])}$. Then $X_{+}$ and $X_{-}$are each homeomorphic to $(S P)_{1}$.

Proof. Since both ends of the compactification are equal to $\Sigma$, the result for $X_{+}$(resp., $X_{-}$) is a consequence of (4.3) and of applying the proof given for (4.6) to an embedding, as in (4.4), of $X_{+}$(resp., $X_{-}$) in the plane.

(4.9) THEOREM. If $X$ is a $C-H$ (4.1.2)-continuum, then $X$ is homeomorphic to $(S P)_{2}$ or $(S P)_{3}$.

Proof. The result follows using (4.8).

We have now proved the first part of (1.1).

In [14] Rogers describes a "wrapping process" to indicate why Cone[ $\left.(S P)_{1}\right]$ and $C\left([S P]_{1}\right)$ are homeomorphic. Rogers also says $[14$, p. 284] that the "wrapping process" for $(S P)_{1}$ may be modified to give a proof that $\operatorname{Cone}\left(S_{0}\right)$ and $C\left(S_{0}\right)$ are homeomorphic. On the basis of what is done in [14, pp. 283-284], it might appear that a modification of the process would yield a similar result for certain other (4.1.1)-continua or for continua such as the ones in Figure 3.2. In various other places in [14] and [15], Rogers asserts that most of the eight continua listed in (1.1) are $\mathrm{C}-\mathrm{H}$ continua; we feel that more detailed proofs of these facts would be helpful. We give such a proof for the case of $S_{0}$; once this is done, our homeomorphism of Cone $\left(S_{0}\right)$ onto $C\left(S_{0}\right)$ can easily be extended and/or modified to give a precise proof for the others.

It is well known and easy to see that an arc is a C-H continuum. To obtain our homeomcrphism of $\operatorname{Cone}\left(S_{0}\right)$ onto $\operatorname{Cone}\left(S_{0}\right)$, we will use the special homeomorphism in (4.10). 
(4.10) Let $I=[0,1]$ and let $\operatorname{Cone}(I)$ be represented by the closed convex hull of the three points $(0,0),(1,0)$, and $\left(\frac{1}{2}, 1\right)$ in the plane. For $(t, 0)$ $\in \operatorname{Cone}(I)$, let $L_{t}$ denote the closed convex arc in $\operatorname{Cone}(I)$ from $(t, 0)$ to $\left(\frac{1}{2}, 1\right)$ :

$$
L_{t}= \begin{cases}\left\{(x, y): y=\left[\frac{2}{1-2 t}\right] \cdot x+\frac{-2 t}{1-2 t}\right\}, & t \neq \frac{1}{2}, \\ \left\{(x, y): x=\frac{1}{2}\right\}, & t=\frac{1}{2} .\end{cases}
$$

Now, let $h: \operatorname{Cone}(I) \rightarrow C(I)$ be defined by

$$
h((x, y))=\{z \in[0,1]:(1-y) \cdot t \leqslant z \leqslant(1-y) \cdot t+y\}
$$

for $(x, y) \in L_{t}$. It is easy to verify $h$ is a homeomorphism of Cone(I) onto $C(I)$.

(4.11) Example (see [14, P. 284]). We show that $\operatorname{Cone}\left(S_{0}\right)$ and $C\left(S_{0}\right)$ are homeomorphic. For conveneince, we use a "piecewise-linear" model $X$ for $S_{0}$ with the helf-line "closing down on" $[0,1]=I$ as in (4.13). Let $h$ denote the homeomorphism for $C(I)$ in $(4.10)$. We now describe a model for $C(X)$ (see (4.15)). For each $x, y \in X$, let $[x, y]$ denote the subcontinuum of $X$ irreducible between $x$ and $y$; also, let $[x, I]$ denote the subcontinuum of $X$ irreducible between $x$ and $I$.

Because of the limited space for figures, we label our descriptions in (2) through (5) below for $n=1,2,3$, and 4 rather than for arbitrary $n$. In (4.14):

(1) $C(I)$ is the solid triangle with vertices $\{0\},\{1\}$, and $I$, where the homeomorphism is $h$.

(2) For each $n=1,2, \ldots$, the solid triangle labelled " $T_{2 n-1}$ " represents the hyperspace of the arc in $X$ from $x_{n}$ to $x_{n+1}$; in each case we assume that the homeomorphism between the solid triangle and the hyperspace mimics $h$.

(3) For each $n=1,2, \ldots$, the "wedged" solid triangle labelled " $T_{2 n}$ " represents, with a natural homeomorphism, all subcontinua of the arc in $X$ from $x_{n}$ to $x_{n+2}$ containing the point $x_{n+1}$. Understanding what we mean by "natural" will perhaps be guided by the following: Consider the horizontal line in (4.14), emanating from the point $I \in C(X)$ to the point $\left[x_{1}, x_{2}\right] \in C(X)$, labelled " $L$ ". The "top part" of the boundary of $T_{2 n}$ is the part of $L$ between $\left[x_{n}, x_{n+1}\right]$ and $\left[x_{n+1}, x_{n+2}\right]$. This part of $L$ represents the subcontinua of $\left[x_{n}, x_{n+2}\right]$ which contain $\left[x_{n}, x_{n+1}\right]$ or $\left[x_{n+1}, x_{n+2}\right]$; the point of $L$ midway between $\left[x_{n}, x_{n+1}\right]$ and $\left[x_{n+1}, x_{n+2}\right]$ corresponds to the continuum $\left[x_{n}, x_{n+2}\right]$.

(4) The arc labelled " $K$ " in (4.14), emanating from $I$ to $X$ represents all those subcontinua of the form $[x, I]$. For each $n=1,2, \ldots$, the convex arc $C_{n}$ from the "top" vertex of $T_{2 n-1}$ to the point $\left[x_{n}, I\right]$ of $K$ represents $\{F \in C(X): F$ $\supset\left[x_{n}, x_{n+1}\right]$ and $F$ is of the form $\left.\left[x_{n}, y\right]\right\}$. For each $n=1,2, \ldots$, the "slanted" convex are $C_{n}^{\prime}$ from the point $\left[x_{n}, x_{n+2}\right]$ of $L$ to the point $\left[x_{n}, I\right]$ of $K$ represents $\left\{F \in C(X): F \supset\left[x_{n}, x_{n+2}\right]\right.$ and $F$ is of the form $\left.\left[x_{n}, y\right]\right\}$. Note 
that, for each $n=1,2, \ldots$, the continua represented by $C_{n}$ are exactly the same as those represented by $C_{n}^{\prime}$ together with the "top part" of the boundary of $T_{2 n}$ from $\left[x_{n}, x_{n+1}\right]$ to $\left[x_{n}, x_{n+2}\right]$. The natural correspondence $\zeta_{n}$, between the two geometric representations of these equal collections of continua, is pictured with arrows in (4.12).

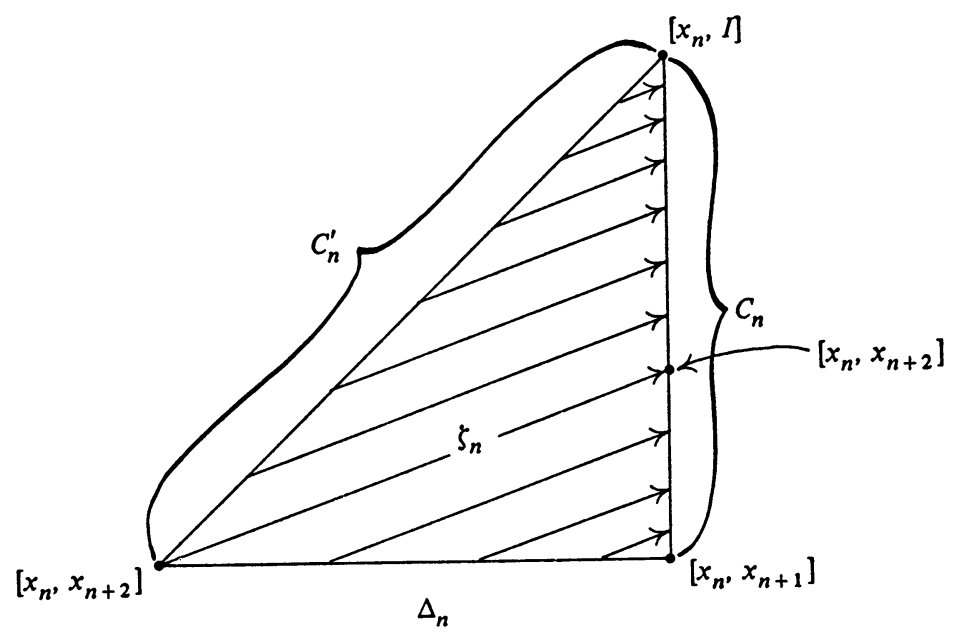

(4.12) FigURE

The homeomorphisms $\zeta_{n}$ will be used later; for this purpose we denote the solid triangle, whose boundary consists of $C_{n}, C_{n}^{\prime}$, and the subarc of $L$ between $\left[x_{n}, x_{n+1}\right]$ and $\left[x_{n}, x_{n+2}\right]$, by $\Delta_{n}$.

(5) Let $x \in X$ be a point on the arc from $x_{n}$ to $x_{n+1}$ (see (4.13)). Then the continuum $\left[x, x_{n+2}\right]$ is represented in (4.14) as a point on $L$ between $\left[x_{n}, x_{n+2}\right]$ and $\left[x_{n+1}, x_{n+2}\right]$. The convex arc going from this point of $L$ to $K$ represents $\left\{F \in C(X): F \supset\left[x, x_{n+2}\right]\right.$ and $F$ is of the form $\left.[x, y]\right\}$; the subcontinuum $[x, I]$ of $X$ is represented by the point of $K$ on this convex arc.

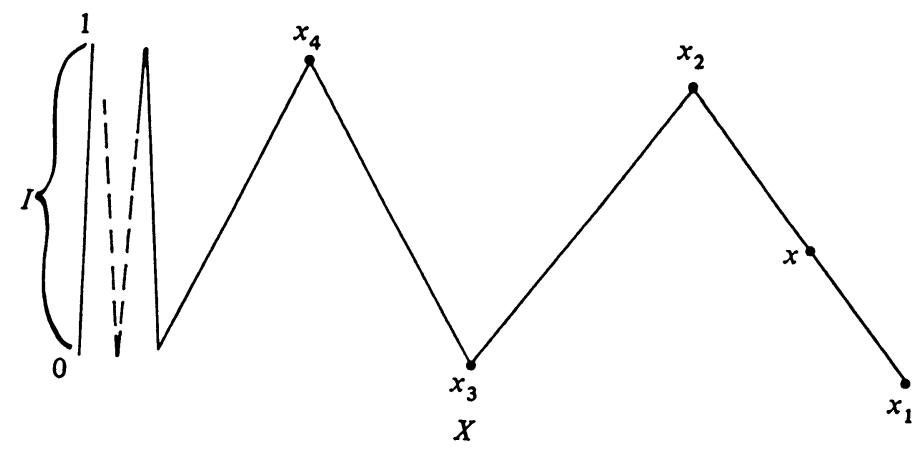

(4.13) FIGURE 


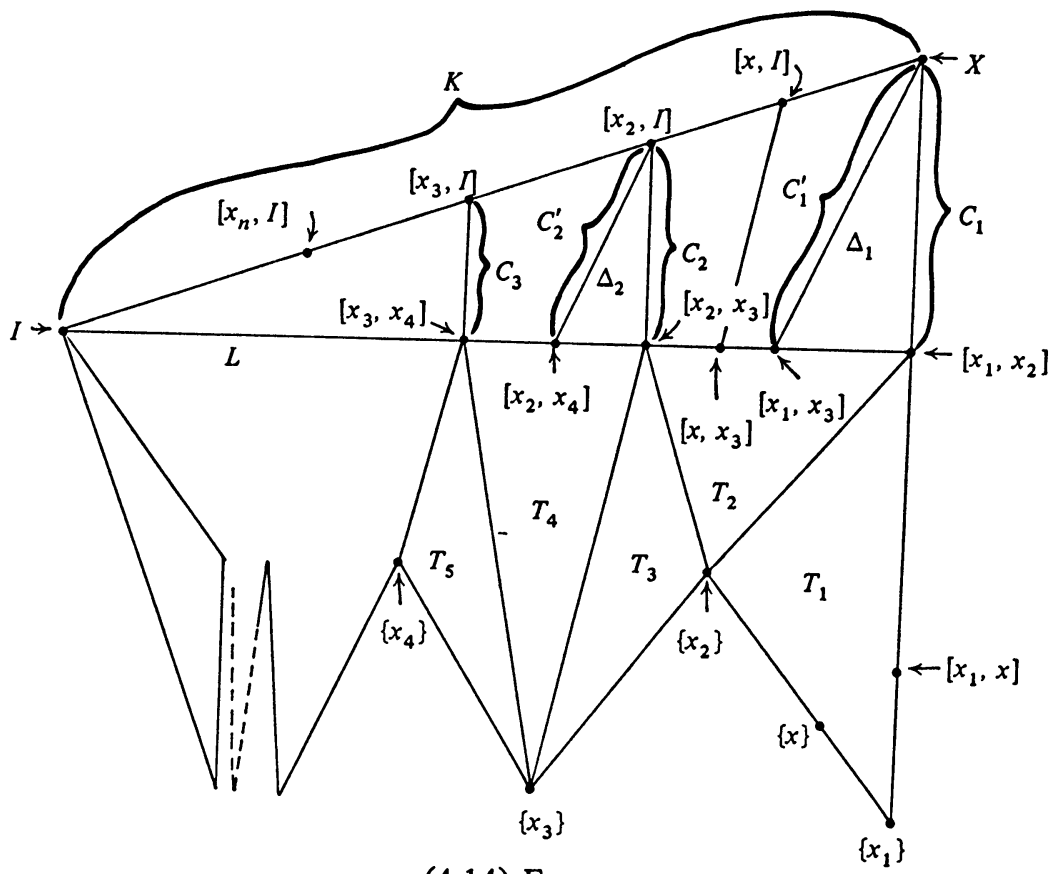

(4.14) FIGURE

Recall from (4) above that the points in the interior of $\Delta_{n}(n=1,2, \ldots)$ do not represent any of the subcontinua of $X$. Also, recall from (4) that, for each $n=1,2, \ldots$, the boundary of $\Delta_{n}$ duplicates some of the subcontinua of $X$ in the manner indicated by the homeomorphism $\zeta_{n}$. Except for these defects, (4.14) represents $C(X)$. Now, for each $n=1,2, \ldots$, identify each point in the domain of $\zeta_{n}$ with its image under $\zeta_{n}$. This amounts to identifying $C_{n}$, for each $n$, in a nice way with the rest of the boundary of $\Delta_{n}$. Since the triangles $\Delta_{n}$ get "smaller and smaller" as $n \rightarrow \infty$, it is easy to see that after making all these identifications, we obtain geometrically the object in (4.15).

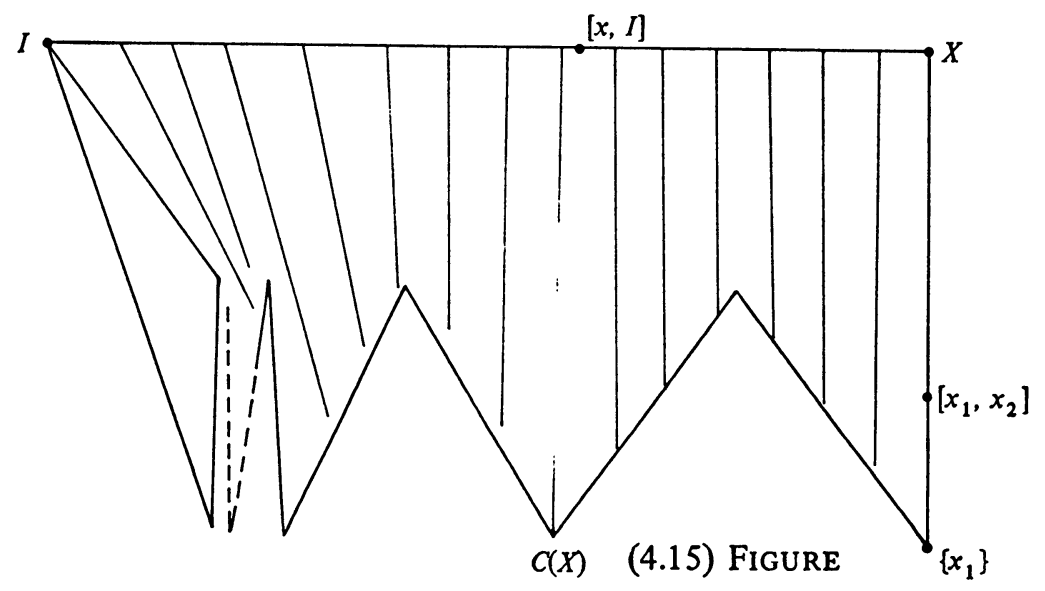


It is clear that the object in (4.15) is homeomorphic to $\operatorname{Cone}(X)$.

5. Indecomposable $\mathbf{C}-\mathbf{H}$ continua. We have now determined the eight hereditarily decomposable $\mathrm{C}-\mathrm{H}$ continua. Next, we focus our attention on the indecomposable $\mathrm{C}-\mathrm{H}$ continua. Our major results begin with (5.4).

We begin with the following lemma, which is a precise formulation of part of a statement in the proof of Proposition 5 of [15] (a misprint occurs in [15, p. 286, line 13 from the top]).

(5.1) LEMMA. Let $X$ be an indecomposable continuum and let $\Lambda$ $\subset[C(X) \backslash\{X\}]$. Then: $\Lambda$ is an arc component of $C(X) \backslash\{X\}$ if and only if there is a composant $\kappa$ of $X$ such that $\Lambda=C(\kappa)$.

Proof. Kelley [5] has shown in the proof of 8.2 that any two subcontinua of $X$ which lie in different composants of $X$ cannot be joined by an arc in $C(X) \backslash\{X\}$. Now, let $\kappa$ be a composant of $X$. Let $D_{1}, D_{2} \in C(\kappa)$. Then, using the indecomposability of $X$, there is a proper subcontinuum $D$ of $X$ such that $D_{i} \subset D$ for each $i=1$ and 2. By [2], $C(D)$ is arcwise connected. Hence, there is an arc joining $D_{1}$ and $D_{2}$ in $C(X) \backslash\{X\}$. The lemma now follows.

(5.2) Lemma. If $X$ is an indecomposable $C-H$ continuum and if

$$
h: \text { Cone }(X) \stackrel{\text { onto }}{\longrightarrow} C(X)
$$

is a homeomorphism, then $h(v)=X$.

Proof. By (1) of (2.1), $C(X) \backslash C(h(v))$ is an open arcwise connected subset of $C(X)$ and, hence, $U=h^{-1}[C(X) \backslash C(h(v))]$ is an open arcwise connected subset of Cone $(X) \backslash\{v\}$. Thus, $\pi[U]$ is an open arcwise connected subset of $B(X)$. Since $B(X)$ is indecomposable, it now follows by [6, p. 212] that $U=\varnothing$, i.e., $h(v)=X$.

(5.3) LemMA. If $X$ is an indecomposable $C$ - $H$ continuum, then each proper subcontinuum of $X$ is contained in some arc component of $X$.

Proof. Let $M$ be a proper subcontinuum of $X$. Suppose that $M$ intersects two arc components $A_{1}$ and $A_{2}$ of $X$. Let $h$ : $\operatorname{Cone}(X) \stackrel{\text { onto }}{\longrightarrow} C(X)$ be a homeomorphism. Since $A_{i}(i=1,2)$ is an arc component of $X$, $\operatorname{Cone}\left(A_{i}\right) \backslash\{v\}$ is an arc component of $\operatorname{Cone}(X) \backslash\{v\}$ and, hence, $h\left[\operatorname{Cone}\left(A_{i}\right) \backslash\{v\}\right]$ is an arc component of $h[\operatorname{Cone}(X) \backslash\{v\}]=C(X) \backslash\{X\}$ by (5.2). Thus, by (5.1) there are mutually disjoint composants $\kappa_{1}$ and $\kappa_{2}$ of $X$ such that $h\left[\operatorname{Cone}\left(A_{i}\right) \backslash\{v\}\right]=C\left(\kappa_{i}\right)$ for each $i=1,2$. Hence, since $B(M) \cap B\left(A_{i}\right) \neq \varnothing$ for each $i=1$, 2, we have that $h[B(M)] \cap C\left(\kappa_{i}\right) \neq \varnothing$ for each $i=1,2$. Therefore, ( $\left.\cup h[B(M)]\right)$ $\cap \kappa_{i} \neq \varnothing$ for each $i=1,2$. Hence, since $\cup h[B(M)]$ is a subcontinuum of $X$ $[5,1.2]$, we have that $\cup h[B(M)]=X$. Therefore, since by (5.2) $X \notin h[B(M)]$, 
we now conclude that $h[B(M)] \cap C(\kappa) \neq \varnothing$ for any composant $\kappa$ of $X$. Thus, using (5.1) and (5.2), we see that $B(M)$ intersects each arc component of Cone $(X) \backslash\{v\}$. Hence, $M$ intersects each arc component of $X$. But, using the fact that composants of $X$ are mutually disjoint [6, p. 212] and the fact that $M$ is a proper subcontinuum of $X$, we see that this cannot be true. The lemma now follows.

The following theorem is a generalization of Theorem 1 of [14] for the indecomposable case.

(5.4) THEOREM. If $X$ is an indecomposable $C$ - $H$ continuum, then each composant of $X$ is arcwise connected. Hence, the composants of $X$ coincide exactly with the arc components of $X$.

Proof. Since $X$ is indecomposable, any arc component of $X$ must be contained in a composant. The theorem is now a simple consequence of (5.3) and the fact that any composant of $X$ is the union of an increasing sequence of proper subcontinua of $X$ [6, p. 209].

(5.5) COROLlary. If $X$ is a finite-dimensional indecomposable $C-H$ continuum, then any composant of $X$ is a one-to-one continuous image of $[0,+\infty)$ or $R^{1}$.

PRoof. The corollary is a direct consequence of (2.5) and the second part of (5.4) together with the fact $[6, p .212]$ that no composant of an indecomposable continuum can be compact.

(5.6) THEOREM. If $X$ is a finite-dimensional indecomposable $C$ - $H$ continuum, then each proper subcontinuum of $X$ is an arc.

Proof. By (5.4), each composant of $X$ is arcwise connected. Thus, we may apply Theorem 5 of [14] to obtain the result.

Our next result shows that finite-dimensional indecomposable C-H continua have a strong form of the cone $=$ hyperspace property (in the sense of [14]).

(5.7) TheOREM. If $X$ is a finite-dimensional indecomposable $C$ - $H$ continuum, then every homeomorphism $h$ : Cone $(X) \stackrel{\text { onto }}{\longrightarrow} C(X)$ takes $v$ to $X$ and $B(X)$ onto X.

Proof. Let $h: \operatorname{Cone}(X) \stackrel{\text { onto }}{\longrightarrow} C(X)$ be a homeomorphism. By (5.2), $h(v)$ $=X$. Hence, we need only show that $h[B(X)]=\hat{X}$. To do this, let $\beta$ be a composant of $X$; by (5.4), $\beta$ is an arc component of $X$. Hence, $\operatorname{Cone}(\beta) \backslash\{v\}$ is an arc component of $\operatorname{Cone}(X) \backslash\{v\}$ and therefore, by $(5.2), h[\operatorname{Cone}(\beta) \backslash\{v\}]$ is an arc component of $h[\operatorname{Cone}(X) \backslash\{v\}]=C(X) \backslash\{X\}$. Thus, by (5.1), there is a composant $\kappa$ of $X$ such that $h[\operatorname{Cone}(\beta) \backslash\{v\}]=C(\kappa)$. By (5.5), there is a oneto-one continuous function 


$$
f_{1}: R^{1} \stackrel{\text { onto }}{\longrightarrow} \kappa \text { or } f_{2}:[0,+\infty) \stackrel{\text { onto }}{\longrightarrow} \kappa .
$$

We prove the following fact:

(5.7.1) If $M \in C(\kappa)$, then there exist real numbers $a \leqslant b$ such that $f_{i}([a, b])=M$ for $i=1$ or $i=2$ (whichever is the case above).

Proof of (5.7.1). Let $M \in C(\kappa)$. For the purpose of proof, assume $M \notin \hat{\kappa}$. First, suppose $f_{i}^{-1}(M)$ is not connected. Then there is a nonempty open interval $(s, t)$ such that $f_{i}^{-1}(M) \cap[s, t]=\{s, t\}$. Clearly, then, $M \cup f_{i}([s, t])$ is a proper subcontinuum of $X$ which is not an arc (because $M \cap f_{i}([s, t])$ $\left.=\left\{f_{i}(s), f_{i}(t)\right\}\right)$. This contradicts (5.6). Hence, $f_{i}^{-1}(M)$ is connected. Thus, since $M$ is an arc by (5.6) and since an arc is not a one-to-one continuous image of a noncompact interval, it now follows that $f_{i}^{-1}(M)=[a, b]$ for some $a$ and $b$. This proves (5.7.1). Using (5.7.1) it is easy to verify the following two statements:

(5.7.2) If $\kappa=f_{1}\left(R^{1}\right)$, then each member of $C(\kappa) \backslash \hat{\kappa}$ is interior to a 2-cell in $C(X)$.

(5.7.3) If $\kappa=f_{2}([0,+\infty))$, then each member of $C(\kappa) \backslash\left[\hat{\kappa} \cup X\left(f_{2}(0)\right)\right]$ is interior to a 2-cell in $C(X)$ [see $\$ 1$ for definition of $X\left(f_{2}(0)\right)$ ]. Also,

$$
X\left(f_{2}(0)\right)=\left\{f_{2}([0, t]): 0 \leqslant t<+\infty\right\} \cup\{X\}
$$

and, hence, $X\left(f_{2}(0)\right)$ is an arc with endpoints $\left\{f_{2}(0)\right\}$ and $X$. Furthermore, $X\left(f_{2}(0)\right) \backslash\left\{\left\{f_{2}(0)\right\}\right\}$ is an open subset of $\hat{\kappa} \cup X\left(f_{2}(0)\right)$.

By (5.6) and (1.2) we have

(5.7.4) No point of $B(\beta)$ is interior to a 2-cell in $\operatorname{Cone}(X)$.

From (5.7.2) through (5.7.4) we now conclude that $h[B(\beta)] \subset \hat{\kappa}$ (note: If $\kappa=f_{2}([0,+\infty))$ then, since each subcontinuum of $B(\beta)$ is nowhere dense in $B(\beta)[6$, p. 207, Theorem 2], it follows from (5.7.3) and (5.7.4) that $\left.\left[h[B(\beta)] \cap X\left(f_{2}(0)\right)\right] \subset\left\{\left\{f_{2}(0)\right\}\right\}\right)$. Thus, since $\beta$ was an arbitrary composant of $X$, it follows that $h[B(X)] \subset \hat{X}$. Therefore, by $(5.6), h[B(X)]=\hat{X}$.

\section{REFERENCES}

1. R. H. Bing, Snake-like continua, Duke Math. J. 18 (1951), 653-663. MR 13, 265.

2. K. Borsuk and S. Mazurkiewicz, Sur l'hyperespace d'un continu, C. R. Soc. Sci. Warsaw 24 (1931), 149-152.

3. G. R. Gordh, Jr. and Sam B. Nadler, Jr., Arc components of chainable Hausdorff continua, General Topology and Appl. 3 (1973), 63-76. MR 47 \#5847.

4. W. Hurewicz and H. Wallman, Dimension theory, Princeton Univ. Press, Princeton, N.J., 1948. MR 3, 312.

5. J. L. Kelley, Hyperspaces of a continuum, Trans. Amer. Math. Soc. 52 (1942), 22-36. MR 3, 315.

6. K. Kuratowski, Topology, Vol. II, English transl., Academic Press, New York; PWN, Warsaw, 1968. MR 41 \#4467.

7. Sam B. Nadler, Jr., Arc components of certain chainable continua, Canad. Math. Bull. 14 (1971), 183-189. MR 46 \#9949. 
8. Continua which are a one-to-one continuous image of $[0, \infty)$, Fund. Math. 75 (1972), 123-133. MR 47 \# 5848.

9. Continua whose cones and hyperspaces are homeomorphic, Notices Amer. Math. Soc. 19 (1972), A718-A719. Abstract \# 72T-G150.

10. Locating cones and Hilbert cubes in hyperspaces, Fund. Math. 79 (1973), 233-250. MR 48 \# 12449.

11. , Multicoherence techniques applied to inverse limits, Trans. Amer. Math. Soc. 157 (1971), 227-234. MR 43 \# 5482.

12. Sam B. Nadler, Jr. and J. Quinn, Embeddability and structure properties of real curves, Mem. Amer. Math. Soc. No. 125 (1972). MR 50 \# 5762.

13. Embedding certain compactifications of a half-ray, Fund. Math. 78 (1973), 217-225. MR 47 \#9544.

14. James T. Rogers, Jr., The cone=hyperspace property, Canad. J. Math. 24 (1972), $279-285$. MR 45 \#4370.

15. Continua with cones homeomorphic to hyperspaces, General Topology and Appl. 3 (1973), 283-289. MR 50 \# 14699.

16. - Dimension of hyperspaces, Bull. Acad. Polon. Sci. Sér. Sci. Math. Astronom. Phys. 20 (1972), 177-179. MR 51 \#6762.

Department of Mathematics, University of Georgia, Athens, Georgia 30602

Current address: Department of Mathematics, University of Kentucky, Lexington, Kentucky 40506 